\title{
SPAG6 silencing inhibits the growth of the malignant myeloid cell lines SKM-1 and K562 via activating $\mathrm{p53}$ and caspase activation-dependent apoptosis
}

\author{
BIHUI YANG, LI WANG, XIAOHUA LUO, LIPING CHEN, ZESONG YANG and LIN LIU
}

Department of Hematology, The First Affiliated Hospital of Chongqing Medical University, Chongqing 400016, P.R. China

Received September 13, 2014; Accepted October 30, 2014

DOI: $10.3892 /$ ijo.2014.2768

\begin{abstract}
SPAG6, which is a novel cancer-testis antigen, is overexpressed in myeloid malignancies. Previously, SPAG6 was found in UPD (uniparental disomy) region of myeloid cell DNA from MDS patients and reported that SPAG6 may be a predictive marker of minimal residual disease in pediatric acute myeloid, but the biological role of SPAG6 in myeloid malignancies remains unclear. The present study was undertaken to determine the expression and functional significance of SPAG6 in malignant myeloid hematologic cell lines. A short hairpin RNA (shRNA) targeting SPAG6 was designed that could specifically inhibit SPAG6 expression at the mRNA and protein levels when introduced into the malignant myeloid hematologic cell lines SKM-1 and K562. The results from flow cytometry and CCK-8 assays showed that SPAG6 silencing inhibited the proliferation of SKM-1/K562 by inducing apoptosis. Furthermore, SPAG6 silencing resulted in activation of caspase- $3,-9$ and -8 and upregulated the mRNA and protein expression of p53 and PTEN. Then, we subcutaneously inoculated the monoclonal cells into NOD/SCID mice to establish xenograft models, and we found that the SPAG6-shRNA lentivirus dramatically inhibited tumor growth and increased apoptosis in vivo. These findings demonstrate that SPAG6 might have a role in malignant myeloid hematologic cell proliferation and apoptosis by regulating caspase proteins and $\mathrm{p} 53$, suggesting that SPAG6 may be a potential therapeutic target.
\end{abstract}

\section{Introduction}

Myelodysplastic syndromes (MDSs) are a group of clonal hematopoietic stem cell disorders characterised by ineffective hematopoiesis leading to blood cytopenias and a variable risk of transformation to acute myelogenous leukemia

Correspondence to: Professor Lin Liu, Department of Hematology, The First Affiliated Hospital of Chongqing Medical University, 1 Youyi Road, Yuzhong, Chongqing 400016, P.R. China

E-mail: liul7776@aliyun.com

Key words: myelodysplastic syndromes, acute myelogenous leukemia, RNA interference, SPAG6, apoptosis
(AML) (1). AML is a hematologic cancer characterised by the accumulation of immature myeloid precursors in the bone marrow and peripheral blood (2), mortality in MDS results from pancytopenia or transformation to AML. Genetic and molecular mechanisms are central in the pathogenesis of hematopoietic diseases such as MDS and AML. It would be of great value for the management of patients with MDS or AML if a number of molecular markers could be identified to enable better prediction of disease progression and prognosis. Recurrent chromosomal abnormalities are detected in approximately half of patients with MDS, the most common single cytogenetic aberrations include del $(5 q)$, trisomy 8 , and del (7q)/-7 (3), and some dominant gene mutations caused by chromosome abnormalities associated with MDS pathogenesis and prognosis have been found (4). High-throughput sequencing technologies and high-resolution whole-genome scanning technologies have revealed molecular alterations in genes of numerous pathways, and the identification of genes relevant to the pathophysiology of malignancy also opens the possibility of targeted therapy. Gene expression profiling studies identified SPAG6 (sperm-associated antigen 6) as an upregulated gene in $\mathrm{CD} 34^{+}$cells from MDS and AML patients (5), hinting that SPAG6 may participate in the occurrence and development of MDS and AML.

SPAG6 was first identified in a human testis cDNA expression library and was named after its function associated with sperm maturation. It has been shown that SPAG6 is a critical protein in the assembly and structural integrity of the sperm tail axoneme and is necessary for flagellar motility $(6,7)$. By immunohistochemical analysis SPAG6 has been found overexpressioned in breast and lung cancer specimens (8). In addition, SPAG6 is overexpressed in pediatric AML and CALM/AF10positive leukemia $(9,10)$, and might thus be the candidate gene to focus on in AML. However, the biological role of SPAG6 in cancers remains vague, the characterization of its effects on hematological malignant cells is still unknown.

In the present study, to clarify the role of SPAG6 in MDS and myeloid leukemia cells, we first confirmed the expression of SPAG6 in hematologic malignant cell lines and then silenced SPAG6 by lentivirus-mediated shRNA in the MDS cell line SKM-1 (derived from a male patient with AML transformed from MDS) (11) and the myeloid leukemia cell line K562 (myeloid blast crisis chronic myelogenous leukemic cells with properties of AML M6) (12). The functional effect 
of SPAG6 was studied through apoptosis, proliferation and cell cycle analysis. In an effort to explain the genetic mechanism by which these effects were obtained, we measured the modulation of the expression of genes known to be involved in apoptosis and oncogenesis. Furthermore, we generated experimental xenografts in NOD/SCID mice to observe the functional effect of knocking down SPAG6 on cell growth in vivo.

\section{Materials and methods}

Cell line culture. The human myelodysplastic syndrome cell line SKM-1, was kindly provided by Professor Jianfeng Zhou from Tongji Medical College of Hua Zhong University of Science and Technology. The erythroleukemia K562, acute promyelocytic leukemia NB4, melanoma A375, liver cancer HepG2, gastric cancer SGC7901 cell lines were kept frozen in our laboratory. Cells line SKM-1, K562, NB4 were cultured in RPMI-1640 and A375, HepG2, SGC7901 were cultured in DMEM, respectively supplemented with $10 \%$ heat-inactivated fetal bovine serum (Gibco, Grand Island, NY, USA) and $100 \mathrm{U} / \mathrm{ml}$ penicillin/streptomycin (Beyotime, Bejing, China) at $37^{\circ} \mathrm{C}$ in a humidified atmosphere of $5 \% \mathrm{CO}_{2}$.

Lentiviral construction and infection. Lentiviral short hairpin RNAs (shRNAs) in pGC-GV vector containing a CMV-driven GFP reporter were obtained from Genechem Ltd. (Shanghai, China). The RNAi target sequence for SPAG6 was 5'-TGATGC TAAATTGAAGCAT-3' and that for nonsense negative control (NC) was 5'-TTCTCCGAACGTGTCACGT-3'. Lentivirus was produced in 293T cells as described previously (13). SKM-1 and K562 cells at exponential stage were plated in 6-well plates $\left(5 \times 10^{4}\right.$ cells/well), transduced with SPAG6-shRNA lentivirus or NC-shRNA lentivirus in the presence of $5 \mu \mathrm{g} / \mathrm{ml}$ polybrene at MOI (multiplicities of infection) of 100 and 20, respectively, as described (14). After 5 days, the transduction efficiency was evaluated by flow cytometry.

Quantitative reverse transcription PCR ( $q R T-P C R)$. Total cellular RNA was extracted from cells using the TRIzol reagent, and the reverse transcription reaction was performed using the Prime Script ${ }^{\mathrm{TM}} \mathrm{RT}$ reagent kit according to the manufacturer's instructions (Takara Biotechnology, Dalian, China). PCR reactions were performed in an ABI PRISM 7500 realtime PCR system (Applied Biosystems, Foster City, CA, USA) for 39 cycles. To normalise for differences in RNA quality and reverse transcription efficiency, we used the reference gene glyceraldehyde-3-phosphate dehydrogenase (GAPDH) as an endogenous control. The relative gene expression levels were quantified using the equation $2^{-\Delta \Delta C t}$. Three replicates of each sample were run on the same plate. The PCR primer pairs used were as follows: SPAG6: F: 5'-CCTTTCAGCTCTCAGTCAG GTTTC-3'; R: 5'-TCTTCACGTTTCATCCTTGTCCTT-3' (product size: 119 bp). GAPDH: F: 5'-CTTTGGTATCGTGG AAGGACTC-3'; R: 5'-GTAGAGGCAGGGATGATGTTCT-3' (product size: 132 bp). P53: F: 5'-TGTGGGATGGGGTGAG ATTTC-3'; R: 5'-CTGTTGGTCGGTGGGTTG-3' (product size: 147 bp). PTEN: F: 5'-ACACGACGGGAAGACAAGTT-3'; R: 5'-CTGGTCCTGGTATGAAGAATG-3' (product size: $157 \mathrm{bp})$.
Western blot analysis. Cells were collected and lysed in RIPA lysis buffer supplemented with $1 \mu \mathrm{M}$ PMSF, and the protein concentration of the supernatant was determined using the BCA protein assay kit (Beyotime). A total of $50 \mu \mathrm{g}$ of protein per lane was boiled in loading buffer, separated by $10 \%$ SDS-polyacrylamide gel electrophoresis (PAGE), and transferred to PVDF membranes. The membranes were blocked with 5\% skim milk for $2 \mathrm{~h}$ and then incubated overnight at $4^{\circ} \mathrm{C}$ with specific antibodies. The following antibodies were used as primary antibodies: rabbit polyclonal antibodies against SPAG6 (Abcam, Cambridge, UK, dilution: 1:1,000); p53 (Immunoway, Newark, DE, USA, dilution: 1:500); PTEN, caspase- $3,-8$ and -9 (Immunoway, dilution: 1:500) and GAPDH (Immunoway, dilution: 1:1,000), $\beta$-actin (Proteintech, Wuhan, China, dilution: 1:1,000) were used as an internal control. After washing with TBS-Tween-20, the membranes were incubated with peroxidase-conjugated goat anti-rabbit $\operatorname{IgG}(\mathrm{H}+\mathrm{L})$ (Proteintech, dilution: $1: 3,000)$ for $2 \mathrm{~h}$ at $37^{\circ} \mathrm{C}$. Excess antibody was removed with TBS-Tween 20 before incubation in ECL. The band intensity was analysed using Quantity One software.

Cell Counting Kit-8 assay for cell proliferation. Cell proliferation was evaluated using a modified CCK- 8 assay after five days of lentivirus infection. Cells in exponential growth were plated in 96-well plates at a final concentration of $2 \times 10^{3}$ cells/well. Briefly, $10 \mu \mathrm{l}$ of CCK-8 (7Sea PharmTech, Shanghai, China) solution was added to the wells, and the plates were incubated at $37^{\circ} \mathrm{C}$ for $2 \mathrm{~h}$. Cell growth was evaluated by measuring the absorbance at $450 \mathrm{~nm}$ using an automated plate reader. All conditions were tested in three replicates.

Cell cycle analysis by flow cytometry. Cells were fixed in $70 \%$ anhydrous ethanol at $4^{\circ} \mathrm{C}$ overnight and stained with $50 \mu \mathrm{g} / \mathrm{ml}$ propidium iodide (PI) containing $50 \mu \mathrm{g} / \mathrm{ml} \mathrm{RNase}$ A for $30 \mathrm{~min}$ at room temperature. Cell samples were analysed using FACSVantage (BD Biosciences, San Jose, CA, USA), and the DNA distribution was analysed using Multicycle software.

Assessment of apoptosis by Annexin $V$ and 7-AAD staining. Cells were harvested, washed twice with ice-cold PBS and resuspended in binding buffer containing $1 \mu \mathrm{l}$ of AnnexinV-PE and $5 \mu \mathrm{l}$ of 7-AAD (KeyGen Biotech, Shanghai, China) (15). All specimens were determined by flow cytometry using CellQuest software (BD Biosciences) after incubation for $15 \mathrm{~min}$ at room temperature in a light-protected area.

Mouse study. To investigate the effects of LV-shRNA targeting SPAG6 on the growth of xenografts, 5-6-week-old male NOD/ SCID mice were purchased from Beijing HEK Bioscience (Beijing, China) and housed within dedicated, individual ventilated cages (IVC) facility at the Laboratory Animal Center of Chongqing Medical University. All animal work was approved by Institutional Animal Care and Use Committee of Chongqing Medical University. The animals were injected subcutaneously with $5 \times 10^{6} \mathrm{SKM}-1 / \mathrm{K} 562$ cells that were stably infected with a lentivirus vector carrying SPAG6-shRNA or with SKM-1/K562 cells stably infected with the NC-shRNA lentivirus.

Twenty-one days after tumor cell inoculation, the mice were sacrificed by cervical vertebra dislocation, and the 


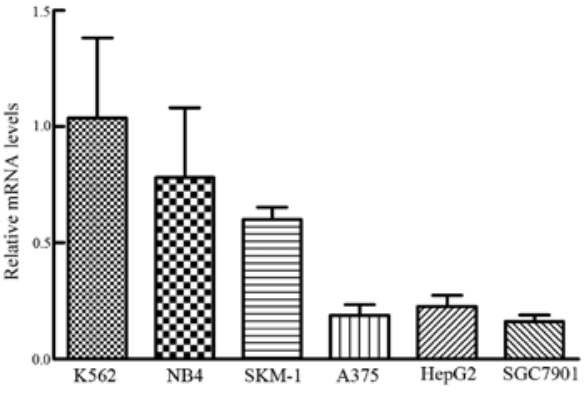

Figure 1. Expression of SPAG6 in the malignant hematologic cell lines. Quantitative RT-PCR showed increased SPAG6 expression in the malignant hematologic cell lines SKM-1, K562, NB4 as compared to positive control HepG2, A375, and SGC7901.

tumoral masses were analysed. The maximum lengths and widths of the xenografts were measured with a calliper, and the tumor volume $\left(\mathrm{mm}^{3}\right)$ was calculated according to the following formula: $0.523 \mathrm{x}$ length $\mathrm{x}$ width ${ }^{2}$ as previously reported (16). Parts of each tumor tissue were embedded in wax, cut into $4-\mu \mathrm{m}$-thick slices, and $\mathrm{H} \& \mathrm{E}$ stained. The apoptosis was detected using terminal deoxynucleotidyl transferase (TdT)-mediated dUTP-digoxigenin nick-end labelling (TUNEL) assays with a commercial TUNEL apoptosis assay kit (Beyotime). The sections were mounted on glass slides, dewaxed, rehydrated through graded alcohols to water, and treated with proteinase $\mathrm{K}$ for $20 \mathrm{~min}$ at $37^{\circ} \mathrm{C}$. Then, the sections were washed with PBS three times and incubated with the $3 \%$ $\mathrm{H}_{2} \mathrm{O}_{2}$ in PBS for 10 min at room temperature. TUNEL assays were then performed according to the manufacturer's instructions.

Statistical analysis. The results are presented as the mean \pm standard deviation. One-way ANOVA and Student's $t$ test were used to examine the significant differences between groups using SPSS 17.0. A value of $\mathrm{p}<0.05$ was considered statistically significant.

\section{Results}

Measurement of the SPAG6 mRNA in hematologic malignancy cell lines. We analysed the expression of the SPAG6 mRNA in the hematologic cell lines MDS SKM-1, erythroleukemia K562, acute promyelocytic leukemia NB4 and in the common solid tumor cell lines melanoma A375, liver cancer HepG2 and gastric cancer SGC7901. Quantitative RT-PCR showed significantly higher expression of SPAG6 mRNA in the hematologic malignant cell lines than in the common solid tumor cell lines $(p<0.05)$ (Fig. 1).

Specific knockdown of SPAG6 expression by lentiviralmediated SPAG6 shRNA. To understand the significance of SPAG6 upregulation in the malignant hematologic cell lines, we used lentivirus expressing shRNA against SPAG6 for gene silencing in SKM-1 and K562 cells. The proportion of infected
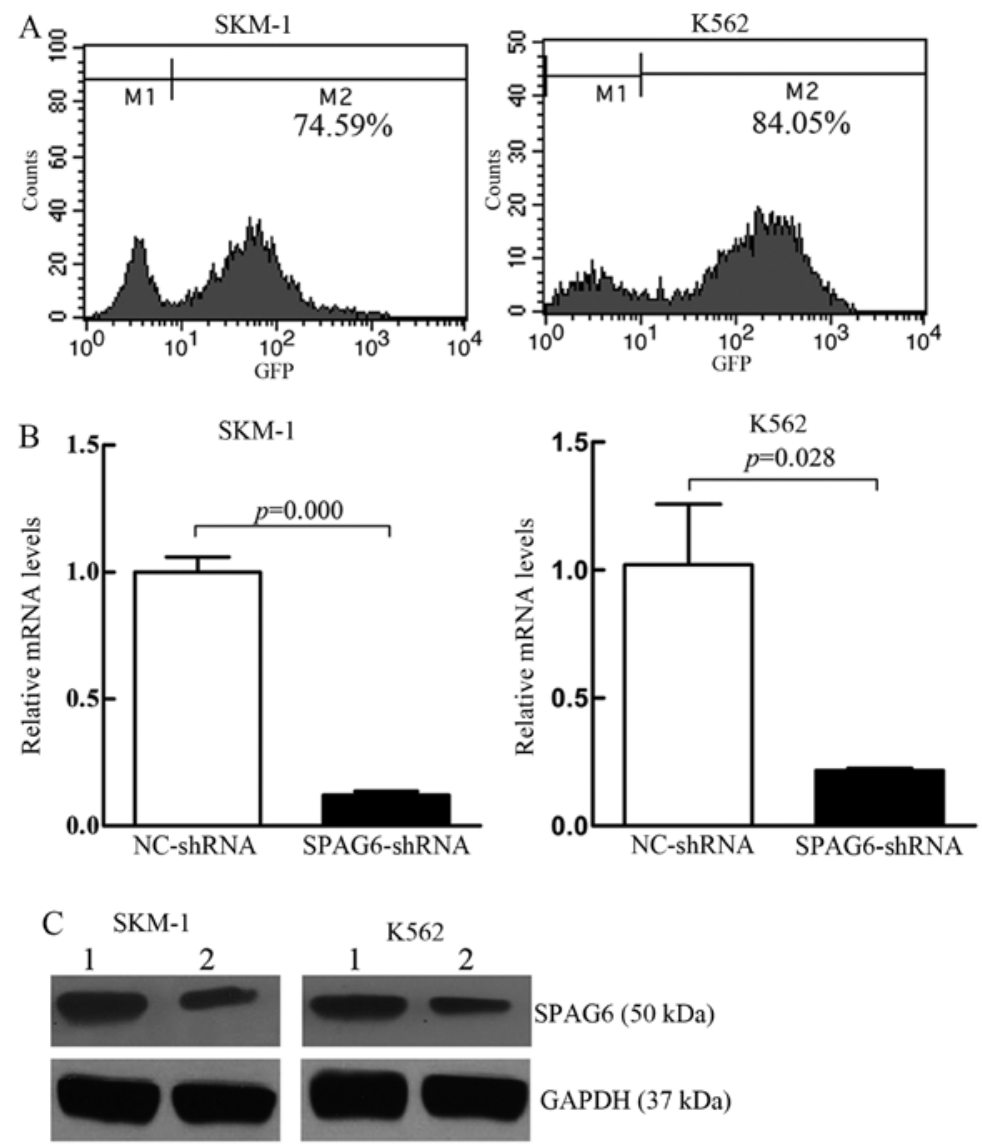

Figure 2. Knockdown the expression of SPAG6 by specific shRNA. (A) Transfection efficiency was detected by FACS. (B) Quantitative expression of SPAG6 mRNA in different groups. Lentivirus-mediated SPAG6 shRNA effectively inhibited SPAG6 mRNA expression both in SKM-1 and K562 cells. (C) Protein expressions of SPAG6 determined by western blot assay using GAPDH as a control for equal sample loading. Lane 1, NC-shRNA group; lane 2, SPAG6-shRNA group. 

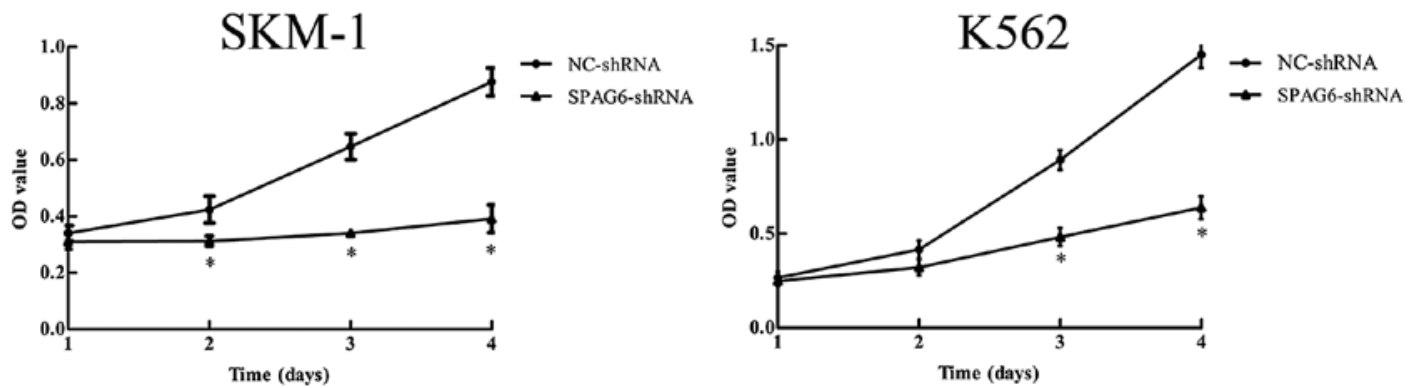

Figure 3. The effect of knockdown of SPAG6 expression on SKM-1 and K562 cell growth. The proliferation of SKM-1 and K562 cells was assessed by CCK-8 assay. The viability of SKM-1 and K562 cells following infection with SPAG6-shRNA lentivirus was significantly decreased.

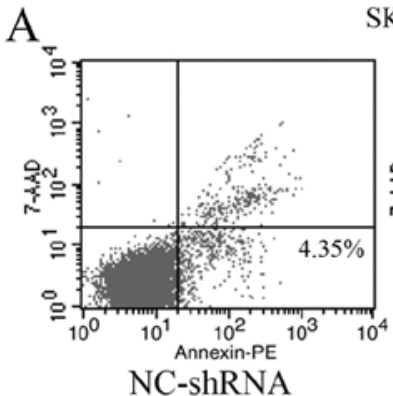

SKM-1

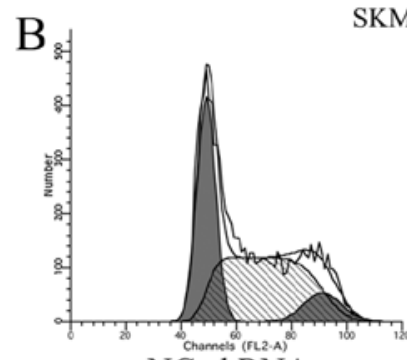

NC-shRNA

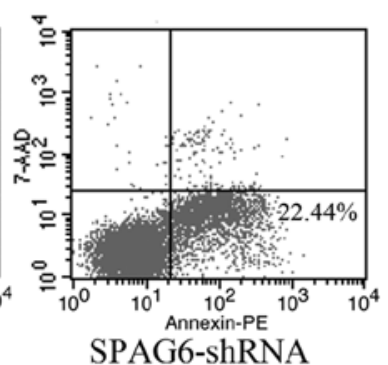

SPAG6-shRNA
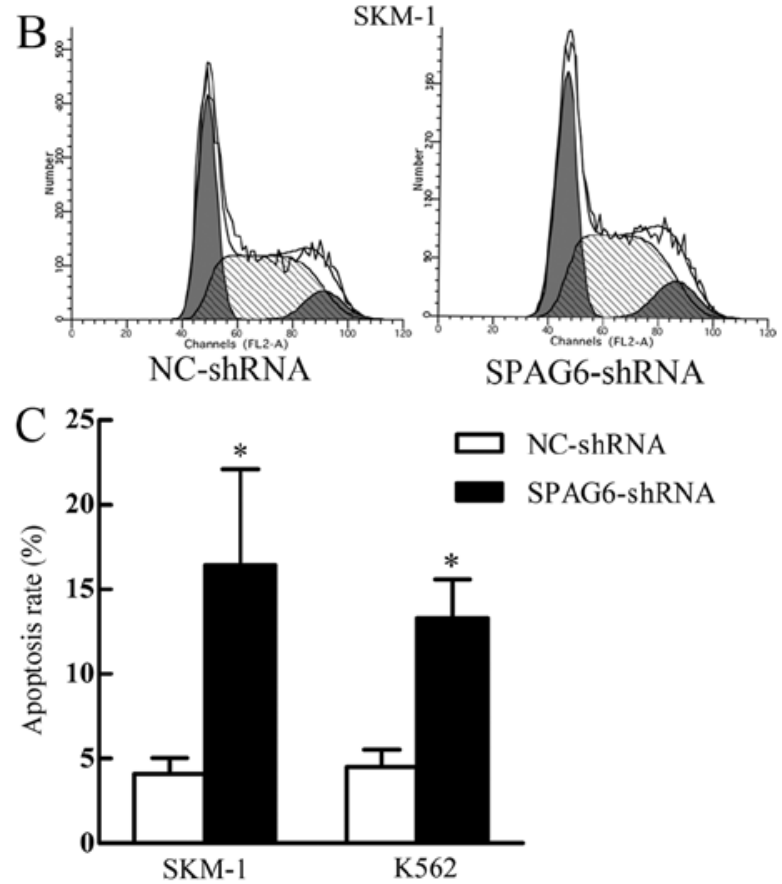

Figure 4. Effect of knockdown of SPAG6 expression on SKM-1 and K562 cell apoptosis and cycle. (A) Apoptosis rate was evaluated by Annexin V and 7-AAD staining after transfection with lentiviral shRNA. (B) Cell cycle was assessed by FACS after transfection with lentiviral shRNA. (C) The analyzed results of the apoptosis rate shown in (A). Apoptosis rate of SKM-1/K562 cells transfected with SPAG6-shRNA significantly increased, when compared with SKM-1/ K562 cells transfected with NC-shRNA ( $<<0.05)$. (D) The analyzed results of the cell cycle shown in (B). The proportion of S phase, G2 and G1 phase in both SKM-1 and K562 cells transfected with SPAG6-shRNA lentivirus showed no significant difference compared to controls.

cells was detected by FACS (Fig. 2A), results showed that the transfection efficiency was $68.53 \pm 5.31$ and $79.36 \pm 5.47 \%$ (Fig. 2B). The transfection efficiencies of the SKM-1 and K562 cells indicated successful infection for suspension cells. The degree of knockdown of SPAG6 expression was determined by qRT-PCR and western blot analysis. The mRNA and protein levels of SPAG6 were downregulated in cells infected with the
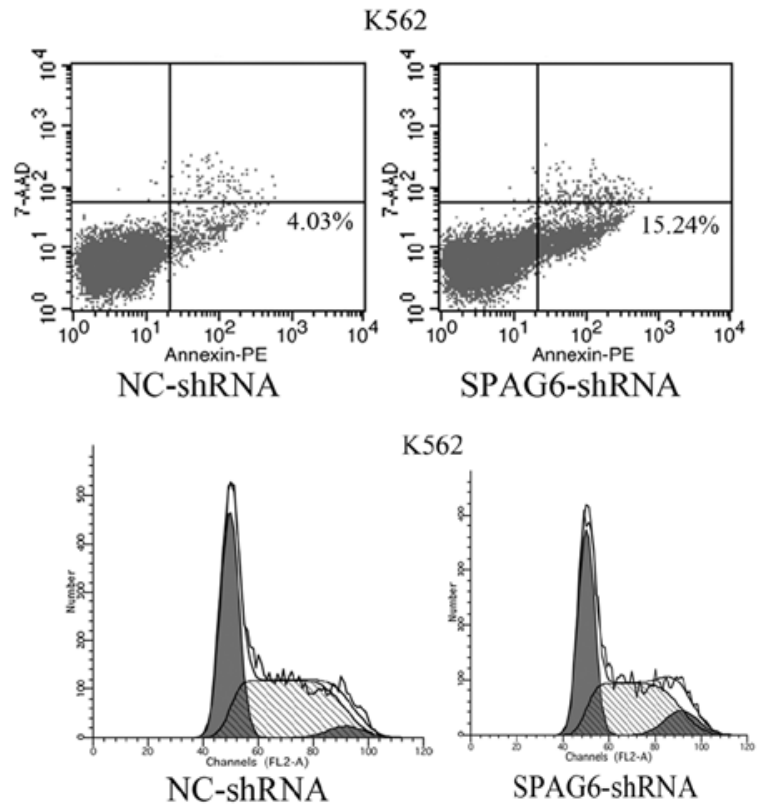

D

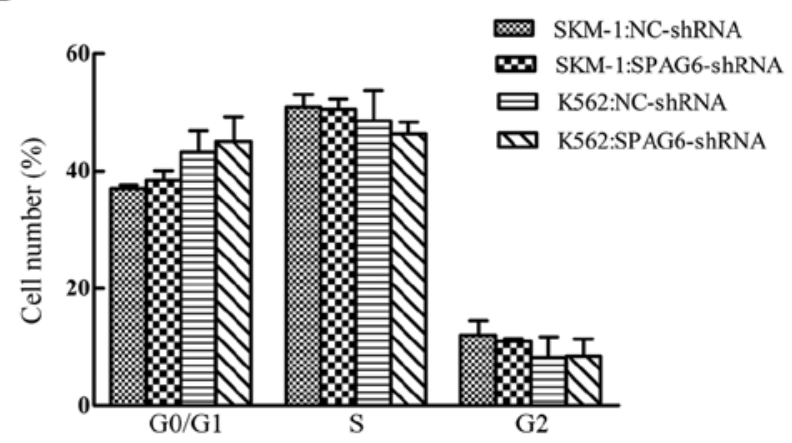

SPAG6 shRNA-lentivirus compared to cells infected with the control lentivirus (Fig. 2B and C).

Effect of downregulated SPAG6 expression on SKM-1 and K562 cell growth. To investigate the possible role of SPAG6 in the growth of SKM-1 and K562 cells, CCK-8 assays were performed to analyse cell proliferation. Cell proliferation was 

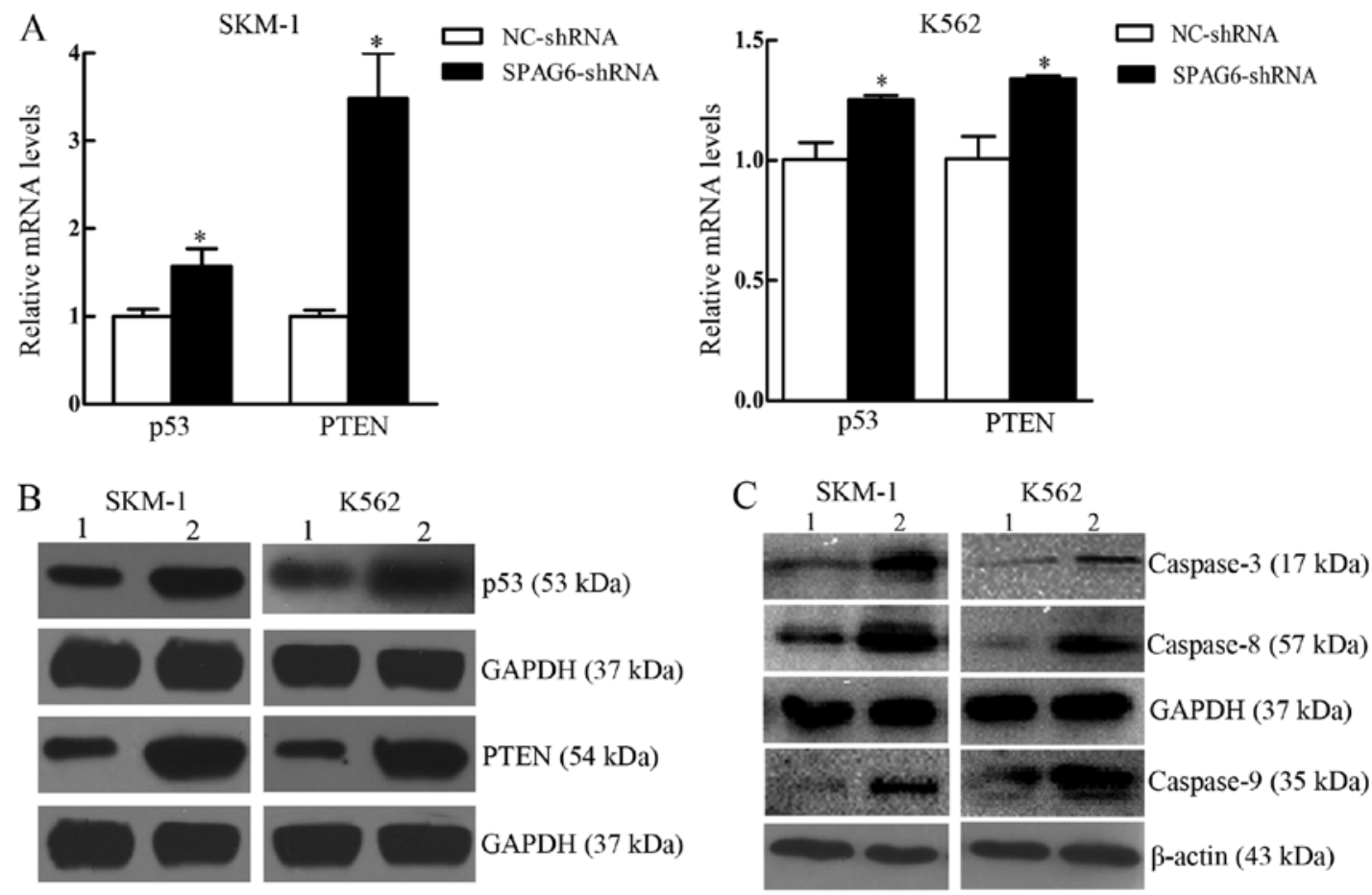

Figure 5. Knockdown of SPAG6 expression increases p53, PTEN expression, and activates caspase-3, -9 and -8. (A) The mRNA expression of the p53 and PTEN were determined by qRT-PCR. "p $<0.05$. (B) Protein expression of $p 53$ and PTEN was determined by western blot assay. (C) The expression of the apoptotic proteins caspase-3, -9 and -8 in different groups. Lane 1, NC-shRNA group; lane 2, SPAG6-shRNA group.

significantly suppressed in cells infected with the SPAG6shRNA lentivirus compared with control cells (Fig. 3).

Analysis of the cell cycle and apoptosis. Annexin $\mathrm{V}$ and 7-AAD assays were performed to analyse apoptosis, as shown in Fig. 4A and C, the percentage of apoptotic cells in the SPAG6 shRNA-infected SKM-1 and K562 cells were significantly higher than in control cells (SKM-1: NC-shRNA 4.12 $\pm 0.52 \%$ vs. SPAG6-shRNA $16.42 \pm 3.27 \%, \mathrm{p}=0.02$; K562: NC-shRNA $4.6 \pm 0.57 \%$ vs. SPAG6-shRNA $13.33 \pm 1.31 \%, \mathrm{p}=0.004)$. In addition, the effect of SPAG6 knockdown on the cell cycle was analysed by flow cytometry, which showed no significant difference in the proportion of cells in S, G2 or G1 phase when SKM-1 cells were infected with the SPAG6-shRNA compared to the controls, and similar results were obtained for K562 cells ( $p>0.05$ ) (Fig. 4B and D).

Knockdown of SPAG6 regulated the expression of apoptotic proteins in SKM-1 and K562 cells. To understand the molecular mechanism by which SPAG6 regulates apoptosis, we examined the expression of apoptotic factors such as $\mathrm{p} 53$, PTEN and caspase-3, -9 and -8 in SKM-1 and K562 cells with SPAG6 knockdown. Quantitative RT-PCR and western blot assays showed the expression of p53 and PTEN at both the mRNA and protein levels was obviously higher in the SPAG6 shRNA-infected cells (Fig. 5A and B). Western blot analysis showed that the protein levels of caspase-3, -9 and -8 were significantly higher in SKM-1 and K562 cells infected with the SPAG6 shRNA lentivirus than the control group (Fig. 5C).

Knockdown of SPAG6 inhibits growth of SKM-1 cells and K562 cells in vivo in NOD/SCID mice. Furthermore, the effects of SPAG6 silencing on SKM-1 and K562 cells were investigated in vivo in NOD/SCID mice. Mice injected with SKM-1/ K562 cells transduced by the SPAG6 shRNA lentivirus developed tumors that were significantly smaller than those in mice inoculated with cells transduced by the NC shRNA lentivirus (Fig. 6A and B). H\&E staining of xenografts inoculated with SKM-1 cells transduced by the SPAG6-shRNA lentivirus and NC-shRNA lentivirus showed necrotic tissue in the center of the tumor, inflammatory cell infiltration, disorderly and irregular tumor cell arrangment, with increased nucleo-cytoplasmic ratio (Fig. 6C). In addition, as shown in Fig. 6D, histological examination of xenografts from SPAG6-shRNA lentivirustreated animals showed a significant increase in apoptosis compared with the NC shRNA lentivirus group (SKM-1: NC-shRNA $18.33 \pm 3.51 \%$ vs. SPAG6-shRNA $48.33 .42 \pm 2.52 \%$, $\mathrm{p}=0.000$; K562: SKM-1: NC-shRNA $20.33 \pm 2.52 \%$ vs. SPAG6shRNA $41.33 \pm 3.51 \%, \mathrm{p}=0.001)$.

\section{Discussion}

SPAG6 is best known for its contribution to the sperm tail axoneme and flagellar motility. Research has shown that SPAG6 promotes differentiation of the inner ear hair cells (17) and is a target gene of the transcription factor S-SOX5 in tissues containing cells with motile cilia/flagella (18), and SPAG6 was identified as a cancer-testis (CT) antigen due to its expression in male germ cells, it was also shown to have the ability to elicit the immune response underlying infertility and was proposed to have oncogenic properties and serologic biomarkers. The category of so-called cancer-testis antigens is one of the most prospective groups of proteins for anticancer immune response activation, as normally, they are expressed 
A
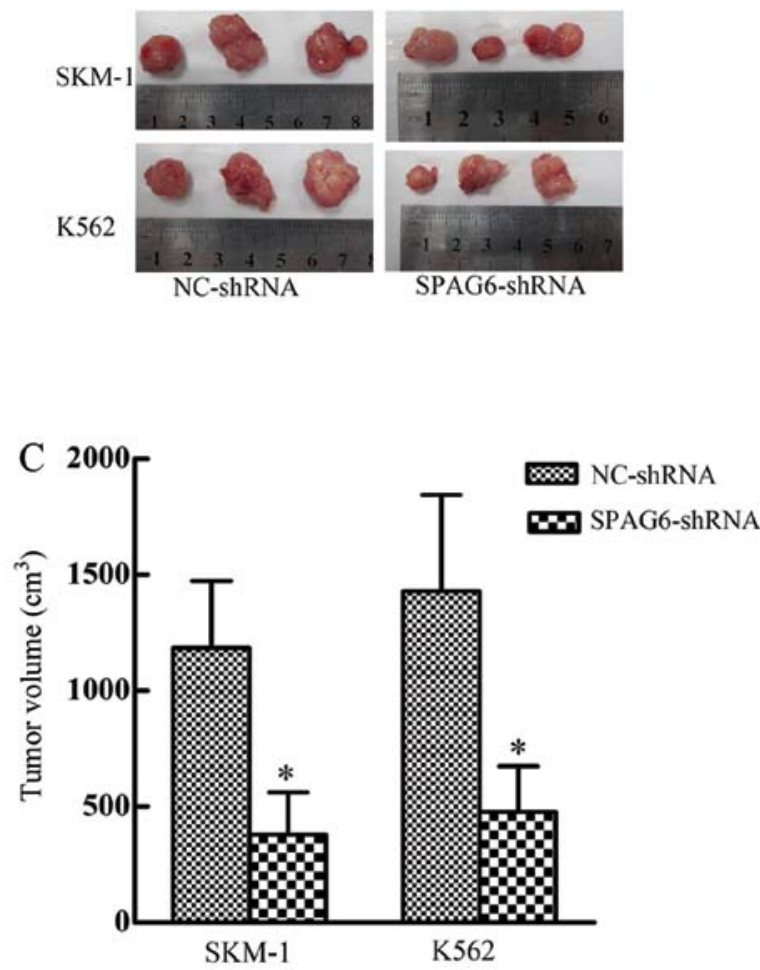

SKM-1
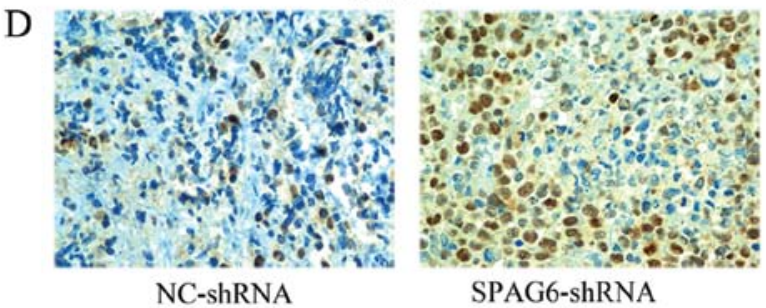
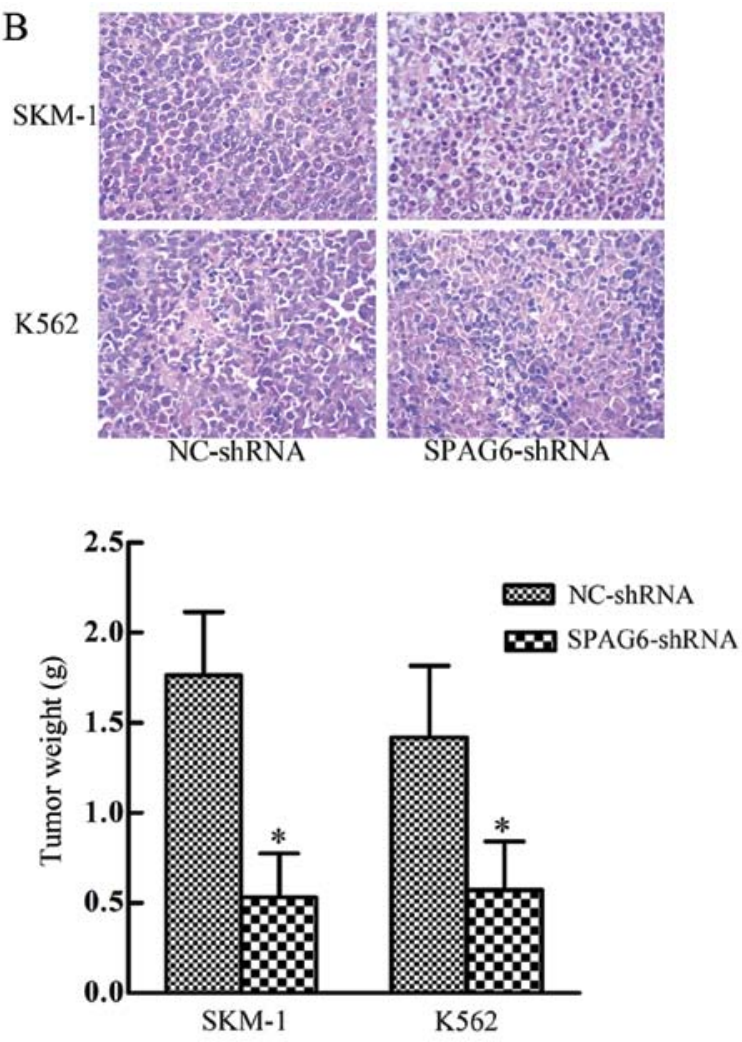

K562

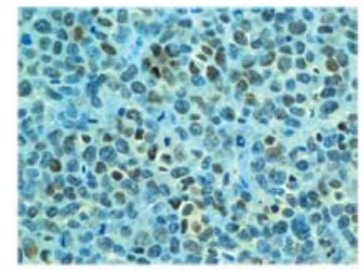

NC-shRNA

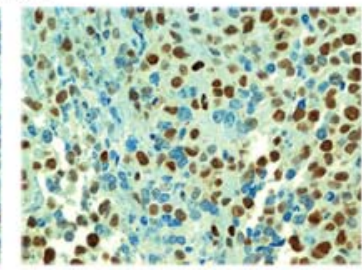

SPAG6-shRNA

Figure 6. The effect of knockdown of SPAG6 expression on SKM-1 and K562 cell growth in vivo. (A) Images of excised xenografts from inoculated mice. (B) Comparison among the volumes and weight of tumors inoculated by SKM-1 and K562 cells treated with different shRNA lentivirus. The tumor size and weight of the group transfected with SPAG6-shRNA lentivirus was significantly decreased compared to that of the control lentivirus transfected cell group $(\mathrm{p}<0.05)$. (B) The H\&E staining of excised xenografts from NOD/SCID mice, showing disorderly tumor cells arranged in with allotypic nuclear division (x400). (C) TUNEL staining showed that in vivo. Cell apoptosis in SPAG6-shRNA lentivirus transfected cell group was significantly increased compared to that in the control groups $(\mathrm{x} 400)$.

in immunologically privileged tissues and are immunogenic if aberrantly generated in tumors $(19,20)$. Therefore, CT antigens have become ideal targets for diagnostics and cancer vaccines. Unlike other family members, SPAG6 has only been identified in a few cancers to date. The upregulation of SPAG6 in cancer cells suggests the possibility that SPAG6 can play a functional role in cancer development.

In our previous study, four recurrent regions of uniparental disomy (UPD) were found in myeloid cell DNA from MDS patients, but not in the T-cell DNA from the same patients. Further study showed that the recurrent UPD at 10p12.31-p12.2 contained seven genes, including SPAG6, and no mutations were identified (21). Interestingly, the expression of SPAG6 in hematologic malignancy is universally significantly higher than in common solid tumors, based on a public encyclopedia (22) (Broad-Novartis. http://www.broadinstitute. org/ccle/home). These data suggest that SPAG6 may be an important factor for the prognosis of hematologic malignancy.
However, its functional role in hematologic malignancy is still not fully clear.

MDS and AML are all myeloid malignancies of the hematologic system, and the low success rate of routine therapeutic methods justifies efforts to develop new approaches. Therefore, in this study, we investigated the functional and transcriptional effects of SPAG6 in MDS and AML using the human MDS cell line SKM-1 and the myeloid leukemic K562 cell line (cells with AML M6 properties) in vitro and in xenografts of NOD/SCID mice in vivo. We transduced human SKM-1 and K562 cells with SPAG6-shRNA lentivirus or control lentivirus. The knockdown efficiency of SPAG6 was validated by qRT-PCR and western blotting following lentiviral transduction, and it was discovered that SPAG6 was significantly downregulated at both the mRNA and protein levels in SKM-1 and K562 cells.

In order to better understand the function of SPAG6 in SKM-1 and K562 cells, we analyzed cell proliferation and 
apoptosis. Apoptosis occurs in a variety of physiological and pathological situations and plays a critical role in maintaining tissue homeostasis in multicellular organisms. The hematopoietic system provides numerous examples attesting to the importance of cell death mechanisms for achieving homeostatic control. Apoptosis may be initiated through different entry sites, such as death receptors or mitochondria, resulting in the activation of effector caspase $(23,24)$. Resistance to apoptosis is one of the hallmarks of human cancers and promotes cancer development and progression (25). In addition, evasion from apoptosis represents one of the leading causes of failure of antileukemic therapy because many anticancer treatments act by triggering apoptosis in cancer cells (26). Our study showed that SPAG6-shRNA treated cells exhibited decreased cell proliferation and increased apoptosis, but the cell cycle was not significantly affected compared to controls. Moreover, antitumor effect of SPAG6-shRNA was observed in vivo, as tumor growth was suppressed and tumor apoptosis was increased in NOD/SCID mice when SPAG6 mRNA and protein were silenced by SPAG6-shRNA. Recently, genome-wide RNA interference screening showed that SPAG6 may be involved in TNF-related apoptosis-inducing ligand (TRAIL)-induced apoptosis (27). In addition, to identify cell division genes in human tissue culture cells, 17,828 initial genes, including SPAG6 were found by genome-scale RNAi profiling, 2,146 final genes, but not SPAG6, altered cell cycle progression, which suggested that SPAG6 may not be involved in cell division (28). Our results are in accordance with previous studies, suggesting that SPAG6 may increase cell growth of SKM-1 and K562 by inhibit cell apoptosis.

Furthermore, we found that knockdown of SPAG6 increased the expression of p53 and PTEN in SKM-1 and K562 cells. As well known, many types of cancers including hematologic malignancies result from functionally impaired p53, the most prominent tumor suppressor, that has lost its capability of DNA repair and apoptosis induction (29). Also, PTEN inactivation is believed to occur frequently in certain hematopoietic neoplasms, the PTEN protein product is a lipid phosphatase that antagonizes PI3K function and consequently inhibits downstream signalling transduction through Akt, and caspase-9 reported to be activated while Akt is inhibited (30). SPAG6 also may be involved in TRAIL-induced apoptosis as mentioned above, TRAIL is a member of the death receptor ligands and can selectively induce apoptosis in tumor cells without severely affecting normal tissue (31). TRAIL can induce caspase- 8 activation and the NF- $\kappa \mathrm{B}, \mathrm{c}-\mathrm{Jun} \mathrm{N}$-terminal kinase and p38 MAPK pathways (32). Therefore, we evaluated the expression of caspase-3, -8 and -9 which are common apoptosis-related genes. In the present study, we found that knockdown of SPAG6 increased the expression of caspase-3, -9 and -8, suggesting that inhibition of SPAG6 expression may be via the PI3K/Akt signaling pathway by increase of PTEN expression and induction of TRAIL-mediated apoptosis, directly or indirectly leading to activation of caspase-3, -9 and -8 , which is responsible for inhibition of SKM-1 and K562 cell growth in vitro and in vivo. Further studies are, however, needed to confirm our results.

In conclusion, the present study demonstrates that SPAG6 is highly expressed in malignant hematologic cell lines. Knockdown of SPAG6 using shRNA potently inhibits proliferation and promotes arrest of apoptosis, but it does not affect the cell cycles of SKM-1 or K562 cells. SPAG6 overexpression may be associated with apoptosis induction via active caspases and increasing p53 expression in SKM-1 and K562 cells. Therefore, SPAG6 is a putative target molecule for drug development aimed to potentiate the effect of apoptosis-inducing drugs. Further investigation of the functional role of SPAG6 may lead to a better understanding of the molecular mechanism of myelodysplastic syndrome and myeloid leukemia. Additionally, the use of drugs suppressing the ability of SPAG6 to induce apoptosis may be an effective strategy for the treatment of myelodysplastic syndrome and myeloid leukemia and therefore need to be explored.

\section{Acknowledgements}

We thank Ms. Juan Du, Ms. Yulin Zhang and Mr. Zhao Liu for their excellent assistance with this project. This study was supported by the Natural Science Foundation of Chongqing (grant no. CSTC2013jjB0145).

\section{References}

1. Cogle CR, Craig BM, Rollison DE and List AF: Incidence of the myelodysplastic syndromes using a novel claims-based algorithm: high number of uncaptured cases by cancer registries. Blood 117: 7121-7125, 2011.

2. Ferrara $\mathrm{F}$ and Schiffer CA: Acute myeloid leukaemia in adults. Lancet 381: 484-495, 2013.

3. Schanz J, Tuchler H, Sole F, et al: New comprehensive cytogenetic scoring system for primary myelodysplastic syndromes (MDS) and oligoblastic acute myeloid leukemia after MDS derived from an international database merge. J Clin Oncol 30: 820-829, 2012

4. Steensma DP and List AF: Genetic testing in the myelodysplastic syndromes: molecular insights into hematologic diversity. Mayo Clinic Proceedings 80: 681-698, 2005.

5. Pellagatti A, Cazzola M, Giagounidis AA, et al: Gene expression profiles of $\mathrm{CD} 34^{+}$cells in myelodysplastic syndromes: involvement of interferon-stimulated genes and correlation to FAB subtype and karyotype. Blood 108: 337-345, 2006.

6. Zhang Z, Jones BH, Tang W, et al: Dissecting the axoneme interactome: the mammalian orthologue of Chlamydomonas PF6 interacts with sperm-associated antigen 6, the mammalian orthologue of Chlamydomonas PF16. Mol Cell Proteomics 4: 914-923, 2005.

7. Zhang Z, Sapiro R, Kapfhamer D, et al: A sperm-associated WD repeat protein orthologous to Chlamydomonas PF20 associates with Spag6, the mammalian orthologue of Chlamydomonas PF16. Mol Cell Biol 22: 7993-8004, 2002.

8. Silina K, Zayakin P, Kalnina Z, et al: Sperm-associated antigens as targets for cancer immunotherapy: expression pattern and humoral immune response in cancer patients. J Immunother 34: 28-44, 2011.

9. Steinbach D, Schramm A, Eggert A, et al: Identification of a set of seven genes for the monitoring of minimal residual disease in pediatric acute myeloid leukemia. Clin Cancer Res 12: 2434-2441, 2006.

10. Mulaw MA,Krause A,Deshpande AJ, et al: CALM/AF10-positive leukemias show upregulation of genes involved in chromatin assembly and DNA repair processes and of genes adjacent to the breakpoint at 10p12. Leukemia 26: 1012-1019, 2012.

11. Nakagawa T, Matozaki S, Murayama T, et al: Establishment of a leukaemic cell line from a patient with acquisition of chromosomal abnormalities during disease progression in myelodysplastic syndrome. Br J Haematol 85: 469-476, 1993.

12. Andersson LC, Nilsson K and Gahmberg CG: K562 - a human erythroleukemic cell line. Int J Cancer 23: 143-147, 1979.

13. Scherr $M$ and Eder M: Gene transfer into hematopoietic stem cells using lentiviral vectors. Curr Gene Ther 2: 45-55, 2002.

14. Wang L, Luo J, Nian Q, Xiao Q, Yang Z and Liu L: Ribosomal protein S14 silencing inhibits growth of acute myeloid leukemia transformed from myelodysplastic syndromes via activating $\mathrm{p} 53$. Hematology 19: 225-231, 2014. 
15. Nian Q, Xiao Q, Wang L, et al: SPARC silencing inhibits the growth of acute myeloid leukemia transformed from myelodysplastic syndrome via induction of cell cycle arrest and apoptosis Int J Mol Med 33: 856-862, 2014.

16. Storti P, Donofrio G, Colla $\mathrm{S}$, et al: HOXB7 expression by myeloma cells regulates their pro-angiogenic properties in multiple myeloma patients. Leukemia 25: 527-537, 2011.

17. Yoon H, Lee DJ, Kim MH and Bok J: Identification of genes concordantly expressed with Atoh1 during inner ear development. Anat Cell Biol 44: 69-78, 2011.

18. Kiselak EA, Shen X, Song J, et al: Transcriptional regulation of an axonemal central apparatus gene, sperm-associated antigen 6 , by a SRY-related high mobility group transcription factor, S-SOX5. J Biol Chem 285: 30496-30505, 2010.

19. van der Bruggen P, Traversari C, Chomez P, et al: A gene encoding an antigen recognized by cytolytic $\mathrm{T}$ lymphocytes on a human melanoma. Science 254: 1643-1647, 1991.

20. Germano S, Kennedy S, Rani S, et al: MAGE-D4B is a novel marker of poor prognosis and potential therapeutic target involved in breast cancer tumorigenesis. Int J Cancer 130 1991-2002, 2012.

21. Wang L, Fidler C, Nadig N, et al: Genome-wide analysis of copy number changes and loss of heterozygosity in myelodysplastic syndrome with del(5q) using high-density single nucleotide polymorphism arrays. Haematologica 93: 994-1000, 2008.

22. Barretina J, Caponigro G, Stransky N, et al: The Cancer Cell Line Encyclopedia enables predictive modelling of anticancer drug sensitivity. Nature 483: 603-607, 2012.
23. Fulda S and Debatin KM: Extrinsic versus intrinsic apoptosis pathways in anticancer chemotherapy. Oncogene 25: 4798-4811, 2006.

24. Hengartner MO: The biochemistry of apoptosis. Nature 407: 770-776, 2000 .

25. Hanahan D and Weinberg RA: Hallmarks of cancer: the next generation. Cell 144: 646-674, 2011.

26. Reed JC and Pellecchia M: Apoptosis-based therapies for hematologic malignancies. Blood 106: 408-418, 2005.

27. Kranz D and Boutros M: A synthetic lethal screen identifies FAT1 as an antagonist of caspase-8 in extrinsic apoptosis. EMBO J 33: 181-197, 2014.

28. Kittler R, Pelletier L, Heninger A-K, et al: Genome-scale RNAi profiling of cell division in human tissue culture cells. Nat Cell Biol 9: 1401-1412, 2007

29. Polager S and Ginsberg D: p53 and E2f: partners in life and death. Nat Rev Cancer 9: 738-748, 2009.

30. Chappell WH, Steelman LS, Long JM, et al: Ras/Raf/MEK/ERK and PI3K/PTEN/Akt/mTOR inhibitors: rationale and importance to inhibiting these pathways in human health. Oncotarget 2: 135, 2011.

31. Ashkenazi A, Holland P and Eckhardt SG: Ligand-based targeting of apoptosis in cancer: the potential of recombinant human apoptosis ligand $2 /$ tumor necrosis factor-related apoptosis-inducing ligand (rhApo2L/TRAIL). J Clin Oncol 26: 3621-3630, 2008.

32. Gonzalvez F and Ashkenazi A: New insights into apoptosis signaling by Apo2L/TRAIL. Oncogene 29: 4752-4765, 2010. 